\title{
UNA APROXIMACIÓN ELSTERIANA DEL TERRORISMO RACIONAL
}

\section{AN ELSTERIAN APPROACH OF THE RATIONAL TERRORISM}

\author{
José Carlos Vázquez Parra* \\ Juan Alberto Amézquita Zamora**
}

\begin{abstract}
RESUMEN
El presente artículo busca hacer un análisis de la racionalidad del acto de terror, señalando las razones que pueden motivar este tipo de atentados. Para llegar a esto, se pretende hacer una aproximación, desde la teoría de la racionalidad elsteriana, la cual recalca la necesidad de hacer un estudio mucho más profundo de la elección misma del actuar, más allá del acto aislado o sus consecuencias. El artículo concluye, entre otros aspectos, que las creencias que respaldan el comportamiento deben ser la pieza central de cualquier aproximación a los actos terroristas y que el sesgo en estos elementos y sus evidencias son determinantes al momento de juzgar la racionalidad o irracionalidad de un acto de terror.
\end{abstract}

PALABRAS CLAVE: TERRORISMO * RACIONALIDAD * CREENCIAS * TERROR * VIOLENCIA

\section{ABSTRACT}

This paper seeks to analyze the rationality of the act of terror and the reasons that motivate such attacks. To achieve this, we intend to make an approach from the elsterian theory of rationality, which stresses the need for a deeper study of the choosing act, beyond the act or its consequences. The article concludes, inter alia, that the beliefs that support the behavior should be the centerpiece of any approach to terrorist acts and that the bias in these elements and their evidence is decisive when judging the reasonableness of an act horror.

KEYWORDS: TERRORISM * RATIONALITY * BELIEFS * TERROR * VIOLENCE

* Departamento de Formación Humanísta y Ciudadana, Tecnológico de Monterrey. jcvazquezp@itesm.mx

** Departamento de Formación Humanística y Ciudadanía, Instituto Tecnológico y de Estudios Superiores de Monterrey.

ja.amezquita@itesm.mx 


\section{INTRODUCCIÓN}

El desarrollo de este intenso siglo xxı ha estado marcado por un sinnúmero de acontecimientos determinantes: desastres naturales, crisis económicas, movimientos sociales, pandemias, despersonalización del individuo, entre otros. Dentro de estos eventos, se resaltan algunos acontecimientos que han dejado una huella profunda de miedo y sangre en lo que se ha avanzado en este nuevo milenio: los actos terroristas.

Aunque el terrorismo no puede ser considerado como un fenómeno plenamente contemporáneo, la configuración de actos de terror que se han presenciado en la última década son muy propios de las necesidades y malestares del mundo moderno. Desde el 11 de setiembre de 2001, con los atentados contra las Torres Gemelas y el Pentágono, hasta los ataques realizados a la revista Charlie Hebdo de Francia el 7 de enero de 2015, hacen que los actos de terror se conviertan en un medio no simplemente para sembrar el miedo y demostrar el alcance de ciertos grupos extremistas, sino que también se ha convertido en un elemento de presión política y principalmente, en un ejemplo de la vulnerabilidad que se tiene como seres humanos.

El terror, el pánico o el miedo extremo paralizan a aquel que lo sufre, incluso lo hacen dudar sobre sus posibilidades de enfrentar estas emociones tan intensas. El miedo vulnera al individuo desde lo más profundo de su ser, generando serios cuestionamientos de su actuar y su sentir (Galimberti 2002). En la actualidad, el miedo se ha convertido en un sentimiento que casi se alberga desde el nacimiento, pues se vive en un mundo sumido en la incertidumbre $y$ no existe mayor temor que pueda tener el ser humano que a aquello que le resulta incierto $y$ desconocido (Vázquez 2013). Por consiguiente, el arma del terror puede configurarse como un elemento que en este milenio toma un nuevo significado, en el que perder la sensación de seguridad, de certeza o protección de parte del Estado puede generar un claro malestar social entre la población.

Sin embargo, aunque la intención de muchos atentados terroristas tiene por móvil la presión política (C. de Gurfinkel 2002), ¿por qué dirigirlas hacía la población civil?, ¿qué es lo que realmente podría motivar a un individuo a realizar un acto de terror?, ¿qué creencias motivan a un agente para este tipo de acciones?, ¿acaso un terrorista concebirá su comportamiento como carente de respaldo? Si llegara a tener argumentos suficientes, ¿se podría decir que un atentado de esta índole puede llegar a considerarse como un acto racional?

El presente artículo busca hacer una aproximación a la posibilidad de un terrorismo racional desde el enfoque de la racionalidad elsteriana, considerando que la propuesta del noruego Jon Elster proporciona el enfoque teórico que más atención pone en los elementos constitutivos de la acción, específicamente a las creencias motivadoras de los actos, lo cual resulta determinante al hablar del comportamiento terrorista.

Por consiguiente, primeramente se hará una breve semblanza de los atentados más significativos de los últimos años, queriendo determinar la existencia de un móvil semejante entre los mismos. Posteriormente, se profundizará en la propuesta elsteriana, planteando un panorama donde sí se analizan los elementos de la acción, con el fin de llegar a comprender la posibilidad de un terrorismo racional.

\section{EL TERROR EN LOS ÚLTIMOS 15 AÑOS}

Aunque parece que una década y media no es suficiente tiempo para modificar la percepción que se tiene de la realidad, este milenio ha demostrado que basta un solo acto para convulsionar la tranquilidad y las creencias de aquello que los individuos perciben como un mundo seguro.

En la mañana del 11 de setiembre de 2001, ninguna persona llegó a imaginar que en pocas horas el mundo cambiaría de una manera radical y que sus vidas serían afectadas, directa o indirectamente, por 19 individuos que tras secuestrar 4 aeronaves comerciales cimbrarían la seguridad de uno de los países más poderosos del mundo. Fueron 2992 personas las que fallecieron con los atentados terroristas perpetrados contra las Torres Gemelas y el Pentágono, entre las 8.00 am y las 10.28 am de ese fatídico día (El Mercurio 2001 y Fox nEws 
2003), sin embargo, el miedo generado no solo afectó a sus familias y al resto de la población de los Estados Unidos, sino que sembró en el mundo una clara sensación de vulnerabilidad, demostrando que un individuo con un acto de terror puede llegar a cambiar la visión del entorno. Actualmente no hay viajero que pueda evitar recordar ese atentado terrorista, en el que dos aviones comerciales fueron convertidos en proyectiles de destrucción masiva. Esto ocasionó que la aeronáutica y sus procedimientos de seguridad nunca volvieran a ser los mismos, pues sencillamente el 11 de septiembre resultó ser un día que cambió el mundo (20min.es, 2015).

En poco tiempo sucedieron otros actos similares de menor escala. El 12 de octubre del 2002, en el distrito turístico de Kuta en la isla indonesia de Bali, detonaron tres bombas que ocasionaron la muerte de 202 personas entre las cuales había 164 extranjeros (Indo 2003). El 16 de mayo de 2003, se dieron los peores atentados terroristas en la historia de Marruecos, cuando una serie de actos suicidas en la ciudad de Casablanca terminaron con la vida de 45 personas (El País 2003). Entre el 15 y el 20 de noviembre de ese mismo año, pierden la vida más de 60 personas en Estambul con una serie de ataques contra algunas sinagogas y el consulado británico (ABC 2006).

El 11 de marzo de 2004, entre las 7.36 a.m y las 07.40 a.m, 10 explosiones casi simultáneas en cuatro trenes de la red de Cercanías de Madrid ocasionaron la muerte de 192 personas (Labari 2004), en un atentado que convulsionaría a Europa Occidental y que lamentablemente se repetiría en el Sistema de Transporte Público de Londres el 7 de julio del 2005 , donde 4 bombas causarían la muerte de otras 56 personas (BBC NEws 2005).

Con el recorrido de estos hechos, se puede llegar a una desalentadora conclusión: las acciones violentas para infundir el miedo en la población son cada día más cotidianas, más sangrientas y más mortíferas.

Uno de los sucesos que daría la vuelta al mundo, es lo ocurrido en la ciudad de París el 7 de enero de 2015, cuando dos hombres enmascarados $y$ armados con rifles de asalto entraron en las oficinas del semanario Charlie Hebdo disparando y matando a 11 personas (Bronstein 2015). Este no únicamente fue un acto repudiado por los medios de comunicación y la población en general, como había sucedido con anteriores atentados, sino que de manera adicional vino a cuestionar el móvil de los actos terroristas. Además del repudio generalizado a tales actos por parte de la población, diversos reporteros, académicos e incluso jefes religiosos acompañaban su rechazo a la violencia con una reflexión adicional en la que cuestionaban si podría justificarse este tipo de acciones como respuesta a una agresión en las creencias de los individuos (CNN México 2015).

A pesar de que el ataque a Charlie Hebdo es tan reprochable como el resto de los atentados terroristas por la utilización de la violencia como alternativa de actuación, en esta ocasión se podría dilucidar aquellas razones que dieron lugar al acto mismo de terror, el cual aunque podrá no tener una justificación moral, puede ser analizado desde una perspectiva que nos revele su posible carácter racional.

Charlie Hebdo es un semanario que en variadas ocasiones fue considerado como excesivo en los comentarios o sátiras que hacía a ciertos grupos políticos o religiosos, con lo cual surge la siguiente interrogante (Stelter 2015): ¿Forman parte de la libertad de expresión, las acciones o la manifestación de las propias ideas en formas que violentan o atentan contra la libertad religiosa o de creencias de otros grupos? e independientemente del acto violento, que no puede ser justificable, ¿si alguien es ofendido, acaso no tiene derecho a replicar la ofensa? Estos cuestionamientos dan pauta a hacer un análisis de la racionalidad del acto terrorista, pues a diferencia de los anteriores atentados que suelen respaldarse simplemente en móviles religiosos o políticos, en esta ocasión es notoria la relación directa del ataque previo de Charlie Hebdo, por la publicación de cartones satíricos en contra de diversas confesiones religiosas, afectando así a aquello que un individuo considera valioso como serían sus propias creencias. El terrorismo es un acto humano y por ende, es factible de ser analizado tanto en el deseo que 
lo genera, las creencias que lo respaldan y la escala de preferencias que dan cabida a la decisión de actuarlo.

\section{LOS ACTOS DE TERROR: SU DEFINICIÓN Y SUS MOTIVACIONES}

Se entiende por acto de terror o terrorismo, según la Real Academia de la Lengua Española (RAE 2015), a la actuación criminal que reiteradamente $y$ de modo indiscriminado, pretende crear una alarma social e infundir terror en la población, principalmente con fines políticos. Según Zuinaga (2011), el terrorismo en la actualidad se presenta como una de las más peligrosas amenazas a nivel global, pero que debe entenderse a partir de dos ópticas, una que define al terrorismo por su finalidad de producir terror $y$ otra que considera que el terror es solo un medio para un fin mayor, que es la presión de carácter político.

El concepto de terrorismo fue utilizado por primera vez por Edmund Burke al hacer referencia al régimen de terror Jacobino durante la Revolución Francesa (Laqueur 2003), sin embargo, la idea principal de la noción hacía referencia al uso de la violencia con fines políticos, lo cual la mayoría de las ocasiones se relacionaba fundamentalmente con agresiones del Estado a un grupo o la población en general. No fue sino hasta después de la Segunda Guerra Mundial que el término terrorismo denota la idea que se tiene actualmente, pues se convirtió en un mecanismo de lucha de los grupos revolucionarios, que buscaban, por medio de actividades violentas, conseguir objetivos políticos.

Según el folleto informativo no. 32 de la Oficina del Alto Comisionado de las Naciones Unidas para los Derechos Humanos (2008), el terrorismo debe considerarse como cualquier acto destinado a causar la muerte o lesiones corporales graves a un civil o a cualquier otra persona que no participa directamente en las hostilidades dentro de un conflicto armado o bien, a aquellos actos criminales con fines políticos concebidos o planeados para provocar un estado de terror en la población en general, en un grupo de personas o en personas determinadas.
De manera complementaria a estas concepciones, teóricos de diversas disciplinas se han dedicado a tratar de comprender las diferentes aristas de este tema, pues consideran que no se puede encasillar al terrorismo únicamente como un medio de presión política, sino que también debe de concebirse como un ataque directo a la población civil.

Friedrich Hacker consideraba que el terrorismo es un mecanismo por medio del cual los débiles y los despreciados conseguían ser tomados en serio y ser escuchados, por lo que planteaba que el móvil fundamental de los actos de terror radicaba en el descontento social con la clase política y la desigualdad entre la población (González 2006). Para Jean-Marie Balencie, el terrorismo tiene la intención de generar un clima de miedo e inseguridad que impresione a la población, ya que no únicamente se configura como un mecanismo de presión, sino también como un medio para influir en las decisiones que se pretenden tomar dentro de un grupo, lugar o tiempo determinado (Rodríguez 2012). Para Della Porta, la intención del terrorista tiene una mayor relación con el concepto que tiene el ciudadano de la autoridad, pues los actos de terror buscan causar un impacto mediático, a través del cual la sociedad entra en pánico y desconfíe de sus gobernantes. Bajo esta concepción, los medios de comunicación resultan determinantes para la consecución del objetivo del acto, el cual se vuelve más relevante en cuanto genere una gran angustia, miedo o terror a un mayor número de individuos.

Por otra parte, De la Corte, Kruglanski, Díaz y Sabucedo (2007) enuncian cinco argumentos o creencias legitimadoras del comportamiento terrorista, entre las cuales se mencionan la crítica a la injusticia social, la identificación de un enemigo institucional, el apego a los valores de grupos extremistas o de insurrección, la búsqueda de alcanzar objetivos comunitarios y la intensión de hacer realidad la idea de un Estado óptimo.

De esta manera, se puede vislumbrar que la intención de un agente para realizar un acto de terror no únicamente se enfoca en factores políticos, como podría resultar en ataques que se dan dentro de las guerrillas o 
movimientos de insurrección, sino que también las razones que explican este tipo de comportamientos pueden recaer en factores mucho más personales como lo serían la influencia social, algunas ideologías e incluso, elementos tan arraigados como las emociones $y$ las creencias propias del individuo.

\section{EL ANÁLISIS RACIONAL DE LA ACCIÓN}

Para poder comprender una actuación, como en este caso se pretende hacer con los actos de terror, es necesario profundizar en las razones que explican dicho comportamiento más allá de la simple intencionalidad, pues cualquier agente, que pueda ser considerado psíquicamente sano, actúa motivado en gran medida por la razón, aunque ello no basta para acreditar que su acción sea realmente -completamente-racional.

Jon Elster (1999) considera que la acción se manifiesta como el resultado de un elaborado proceso reflexivo, en el que se debe tomar en cuenta todos los elementos constitutivos del acto. En un primer momento, se debe cerciorar de que existe una relación congruente entre los deseos que motivan a actuar y las creencias que permiten generar alternativas de actuación. Después, como agentes racionales, se debe analizar que tanto los deseos como las creencias se encuentren argumentadas con evidencia suficiente y óptima, pues solo así se podra señalar como elementos objetivos $y$ apegados a la realidad. Cuando se consigue esto, se podría realmente acercar a una explicación de los actos desde la perspectiva elsteriana, considerando los deseos que nos mueven y las creencias que los respaldan (Vázquez 2012).

Sin embargo, el entender la acción, no justifica realmente que esta sea racional, pues poder decir que una acción cuenta con esta característica, desde la perspectiva elsteriana, implica un proceso reflexivo mucho más detallado. Para Elster (2010), un acto racional debe cumplir tres condiciones: a) la existencia de un conjunto de alternativas posibles; b) que el agente tenga un grado de certidumbre sobre el resultado de cada una de las acciones del conjunto $y, c)$ que el agente pueda dar una medida cuantificable de cada una de las alternativas propuestas. El filósofo noruego pone especial atención en estos tres momentos del acto, pues considera que la escala de preferencia que se hace antes de elegir como actuar, es determinante para saber si la actuación es realmente la mejor opción para cumplir los fines señalados por los deseos.

Por lo anterior, un punto que se vuelve primordial en esta percepción elsteriana es el del papel que desempeñan las creencias al fundamentar las opciones de actuación, ya que es importante considerar que no se puede dar una acción sin una elección previa y no se puede llegar a elegir en aquella situación en que no se tengan por lo menos dos opciones que se crean óptimas y convenientes para estos fines. La certidumbre que se puede llegar a tener de cada una de las alternativas de actuación se logra en gran medida a partir de la recolección de evidencia óptima que respalde las creencias, pero aun así, aunque la incertidumbre se reduzca, Elster reconoce que la previsión de las consecuencias de los actos es simplemente un ideal. Se debe resaltar que para que las alternativas de actuación y por ende, la elección, sean realmente racionales, es necesario que dichas creencias, aunque nunca dejen de ser inciertas, se encuentren debidamente fundamentadas, pues Jon Elster considera que solamente será a partir de la relación que existe entre las creencias y la evidencia que se posee acerca de ellas, estas pueden ser consideradas racionales $y$ por ende, cercanas a la realidad (Arredondo y Vázquez 2013).

De tal manera y con base en todo lo anterior, se puede decir que para llegar a una acción racional, el proceso natural sería elegir entre las alternativas que se poseen, aquella que resulta óptima, considerando que tal evaluación se da conforme a nuestra escala de preferencias, la cual se estructura en gran medida por aquello que se cree, permite acercarse lo más posible a la satisfacción del deseo (Vázquez 2015).

Las consideraciones anteriores permiten comprender por qué se está obligado a prestar una especial atención a las creencias, pues estas resultan ser un tema determinante de ser explicado y comprendido al momento de querer analizar una actuación. Las creencias son la base 
de las alternativas de acción que se prevé realizar, así que si nuestro comportamiento se basa en creencias irracionales, es obvio que dé como resultado, decisiones igualmente irracionales, que aunque para nosotros tengan sentido, resultan desconcertantes para los observadores externos.

Así, antes de poder valorar un acto desde cualquier perspectiva, es necesario que se profundice en las creencias que respaldan al individuo que actúa, pues es complicado valorar la racionalidad del comportamiento sin antes evaluar la racionalidad de las creencias del agente.

\section{LAS CREENCIAS TERRORISTAS}

Cuando se habla de un terrorista, comúnmente se cae en la postura de considerar que lo definen dos rasgos que no siempre son los más apegados a la realidad: un individuo poco educado, que carece de autonomía, porque se encuentra influido por un grupo de presión o de extremismo religioso, o bien, una persona que padece de un desorden antisocial. En cualquiera de estas dos opciones se hace referencia a una persona que actúa fuera de la razón. Sin embargo, ¿qué tanto se puede decir respecto a que un acto de terror sea irracional?

Durante la Conferencia Internacional de las Naciones Unidas sobre Financiamiento para el Desarrollo del 2002, el presidente de Brasil, Luiz Inácio Lula da Silva, hizo una declaración que todavía hoy mantiene su vigencia, al señalar que la solución al terrorismo radicaba más en el alivio a la pobreza que el diseño de estrategias militares (Pavón y Velázquez 2006). Sin embargo, aunque pudiera haber una relación causal entre pobreza y terrorismo, no se puede considerar que esta explicación sea suficiente, pues la justificación resulta de un argumento fácilmente cuestionable (Krueger 2003).

Después de los atentados del 11 de setiembre en Nueva York, algunos periodistas se dieron a la tarea de investigar el perfil de los individuos que realizaron el ataque, con lo cual descubrieron que estos eran considerados por sus vecinos como individuos educados, atentos y cordiales. Específicamente, dos de los terroristas habían estudiado ingeniería electrónica en la Universidad Tecnológica de Hamburgo, en
Alemania, estaban casados, tenían hijos, bebían alcohol y hablaban varios idiomas (Barón 2001).

Pavón y Velázquez (2006), consideran que más que atribuir el terrorismo a una situación de pobreza o a la pertenencia a un estrato socioeconómico bajo, el terrorismo se explica por causas muy variadas, entre las cuales se encuentra un claro sentido de injusticia, un alto nivel de compromiso político con una causa y la existencia de un conjunto de valores supremos que desencadenan pronunciamientos y conductas extremas, como puede ser una ideología política o religiosa. C. de Gurfinkel (2002), completa la panorámica anterior al plantear que la educación forma parte fundamental de los procesos de estructuración de valores, que al ser el resultado de un proceso de carencia formativa, pueden gestar ideales absolutos que se contrapongan a nociones como la empatía, el respeto y la tolerancia. De igual manera, Sequera (2001) respalda la idea de que la educación resulta un factor relevante al querer explicar las motivaciones o razones del acto de terror, aunque considera que la educación contemporánea al tener un claro corte social, podría resultar como el mejor método para revertir este tipo de comportamientos extremos, pues la formación en las aulas tiende a la transmisión de valores universales como la igualdad, la libertad, la tolerancia y el respeto al otro, principios que no comparten los actos de terror.

Bajo la perspectiva de la racionalidad del acto, se puede identificar que el comportamiento violento o agresivo se configura como una más de las alternativas de actuación que suele tener la misma validez con la que se actúa de manera amorosa, alegre o responsable, es decir, el acto de terror no es más que el apego a una acción que dentro del proceso racional se ha considerado como el acto óptimo en la escala de preferencias que se ha constituido (Vázquez 2015). De este modo, el móvil de los actos de terror radica en algo mucho más profundo que la toma de decisiones, se ubica más bien en la constitución misma de la escala a partir de creencias que muchas ocasiones no son óptimas.

Para Elster (2010), un comportamiento es racional cuando se basa en dos elementos: un deseo coherente, consistente y autónomo, 
$y$ en un conjunto de creencias respaldadas por evidencia óptima. Si alguno de estos elementos falla, el acto es fácilmente cuestionable. Bajo una perspectiva de racionalidad simple se podría decir que el acto de terror llega a ser racional con el simple hecho de que se sustente en razones suficientes, sin embargo, esto no es correcto en el enfoque elsteriano, pues si al analizar dichas razones se observa que las mismas son irracionales, el acto resulta notoriamente sesgado.

Un comportamiento terrorista que es motivado por ideologías o ideales poco comprensibles o desconocidos para el agente, ya sea por su nivel educativo o motivaciones ocultas, es un claro caso de falta de autonomía, lo cual genera un comportamiento irracional. Asimismo sucede si se sustenta en creencias con muy poco o nulo respaldo, o bien, influenciadas por factores que afectan su objetividad, pues el agente decide con base en lo que cree que es un comportamiento correcto para los fines que persigue, lo que si se sustenta en creencias erróneas, podría resultar en un acto contradictorio a lo que se propone. El análisis de la racionalidad de los actos de terror obliga a profundizar no solo en el deseo que motiva la acción, sino también a la estructura de las alternativas de actuación entre las que el comportamiento terrorista fue considerado la mejor opción.

De esta forma, se puede llegar a comprender $y$ justificar los planteamientos que señalan a la pobreza o a la carencia educativa como factores que pueden llegar a ser determinantes del comportamiento terrorista, no tanto porque sean razones motivadoras suficientes, sino más bien por el lugar que desempeñan en la constitución de deseos autónomos y la generación de creencias racionales. La perspectiva de la racionalidad elsteriana permite enfocar la atención del análisis de la acción en los elementos constitutivos de la misma, más allá de la intención, el motivo y las razones.

\section{LA POSIBILIDAD DE UN TERRORISMO RACIONAL}

Al inicio de este artículo se han planteado algunos cuestionamientos, los cuales fundamentalmente, giran en torno a la posibilidad de un acto racional de terror. Pues bien, ¿podríamos decir que un acto terrorista puede llegar a considerarse como un acto racional?

Desde un enfoque de racionalidad simple, en la que se puede decir que hay racionalidad donde hay razones, la respuesta tendería a ser positiva, pues efectivamente se ha demostrado que en su mayoría, los terroristas que perpetran este tipo de acciones son motivados por razones suficientes para sustentar sus actos. Sin embargo, si se hace el análisis desde un enfoque de racionalidad ampliada como la propuesta por Jon Elster, es claro que habría mucho más que preguntarse para poder decir que un acto de terror alcanza el calificativo de racionalidad.

La posibilidad de un acto terrorista racional radicaría fundamentalmente en la satisfacción, por parte del agente, de los siguientes elementos de actuación:

a) El deseo que motiva la acción es autónomo $y$ no se ve afectado por ningún tipo de presión política, social, religiosa o incluso, motivado por sus emociones o pasiones respecto a un tema.

b) La elección que se toma corresponde a la mejor alternativa enunciada en una escala de preferencias en la que cada una de las opciones de actuación ha podido ser cuantificada conforme al nivel de satisfacción posible del deseo motivador.

c) Las preferencias constituyentes de la escala, así como la elegida, se encuentran fundamentadas en creencias racionales, es decir, que se respaldan por un cúmulo considerable de evidencia óptima, suficiente, objetiva $y$ que no se ha visto menguada por las emociones o creencias personales del agente.

d) La elección racional generada se encuentra relacionada proporcionalmente con la decisión tomada y con el acto realizado, por consiguiente, el proceso de actuación no se ve afectado por factores internos o externos del agente, como sería el caso específico de la debilidad de la voluntad.

Solo de esta forma se puede decir que el acto realizado es objetivamente racional, lo cual, incluso para el mismo Elster, es pocas 
veces alcanzado, pues siempre hay posibilidades de ser irracional. John Rawls en el prefacio de su obra Teoría de la justicia (1997) señala algo semejante al referirse al valor del conocimiento que se alcanza después de una búsqueda diligente: "la medida de mis deudas no es lo corto que he quedado respecto a lo que pudo ser, sino la distancia recorrida desde los comienzos". De tal manera, la racionalidad de los actos no es tanto por lo que se consigue al final, sino más bien por el proceso constitutivo del actuar, es decir, la buena fundamentación de las creencias que los respaldan. Como se ha precisado, no hay acto racional, si se fundamenta en creencias irracionales.

Al referirse a la posibilidad de un acto de terror racional, no se identifica con querer justificar racionalmente el comportamiento terrorista, sino más bien enfatiza la necesidad de hacer un análisis mucho más profundo $y$ específico de los elementos constitutivos de la acción y de la elección misma de actuar en dicho sentido. Aunque un agente cuente con las creencias que sustenten que el acto de terror resulta como una respuesta racional a los requerimientos de cierto deseo, esto no implica que dichas creencias sean realmente racionales, lo que puede cuestionar severamente su presunción de racionalidad.

Para un individuo que ha sido formado a partir de creencias erróneas o basadas en conceptos que no permiten discusión alguna como la fe, la lealtad o el deber, sus comportamientos pueden ser claramente vistos como actuaciones racionales, pues efectivamente tienen razones suficientes para actuar en dicho sentido, aunque dichas razones sean sesgadas e irracionales.

Por consiguiente, el que un agente realice un acto terrorista con la plena creencia de que este es el comportamiento óptimo por contar con razones que considera suficientes para hacerlo no lo acredita como un comportamiento racional, pues el análisis de su actuar no radica en que justifique el comportamiento, sino en que esté basado en creencias realmente óptimas y por ende, racionales. Además, el que las creencias tengan un sesgo estructural amplía la posibilidad de incertidumbre en sus consecuencias, ya que entre más alejadas estén las creencias de la realidad, mayor es la factibilidad de que desencadenen resultados no esperados o incluso, contrarios a lo que se pretende.

En conclusión, el análisis elsteriano de la racionalidad de la acción permite vislumbrar que las razones que motivan nuestros actos no siempre son buenas u óptimas razones, sino que en la mayoría de los casos, son simplemente creencias, que se ven afectadas por factores internos o externos del agente. El comportamiento terrorista es un ejemplo de esto, pues aunque parece conquistar un objetivo a partir de lo que plantea como una acción correcta, simplemente hace que el agente se aferre a creencias irracionales, con un sustento sesgado y con consecuencias peligrosamente inciertas.

\section{REFERENCIAS}

\section{LIBROS}

Elster, Jon. 1988. Uvas Amargas. Sobre la subversión de la racionalidad. Barcelona: Peninsula.

Elster, Jon. 1997. Estudios sobre la racionalidad. Lectura pronunciada en la ceremonia en que se le concedía el título de Doctor Honoris Causa por la Universidad de Valencia. Valencia.

Elster, Jon. 1999. Juicios Salomónicos. Barcelona: Gedisa.

Elster, Jon. 2010. La explicación del comportamiento social: mas tuercas y tornillos para las ciencias sociales. Barcelona: Gedisa.

Galimberti, Umberto. 2002. Diccionario de psicología. Ciudad de México: Siglo XXI editores.

González, Eduardo. 2006. El fenómeno terrorista. Madrid: Dastin.

Laqueur, Walter. 2003. Una historia del terrorismo. Madrid: Paídos.

Rawls, John. 1997. Teoría de la Justicia. México: Fondo de Cultura Económica.

Vázquez, José Carlos. 2013. La incertidumbre en que vivimos. México: Panorama. 


\section{PUBLICACIONES PERIÓDICAS}

20 minutos. 2008. "11-S, el día que cambió el mundo: siete años del mayor atentado terrorista de la historia". 20 minutos, 11 de setiembre. Acceso el 06 de febrero de 2015. http://www.20minutos.es/ noticia/410921/0/aniversario/11-S/nuevayork/

ABC. 2006. "La amenaza terrorista". $A B C$, ¿fecha del documento? Acceso el 06 de febrero de 2015. http://especiales.abc.es/2006/ amenaza-terrorista/

Arredondo, F. G. y Vázquez, J. C. 2013. "Un modelo de análisis racional para la toma de decisiones gerenciales desde una perspectiva elsteriana". Revista Cuadernos de Administración XXVI, n. ${ }^{\circ}$ 46: 107-133.

Barón, A. 2001. "Terroristas suicidas de clase media $y$ alto grado de instrucción”. $E l$ Clarín, 16 de Septiembre. Acceso el 06 de abril de 2016. http://edant.clarin.com/ diario/2001/09/16/i-01215.htm

British Broadcasting Corporation (BBC NEWS). 2005. "London bombings: Police updates". BBC NEWS, 8 de Julio. Acceso el 06 de abril de 2016. http://news.bbc.co.uk/2/hi/ uk_news/4664419.stm

Bronstein, Scott. 2015. "El camino hacia el terrorismo de los atacantes de Charlie Hebdo". CNN México, 15 de enero. Acceso el 06 de abril de 2016. http://mexico. cnn.com/mundo/2015/01/14/el-caminohacia-el-terrorismo-de-los-atacantes-decharlie-hebdo

C. de Gurfinkel, Laura C. 2002. "Fundamentalismo, terrorismo y educación". Educere 5, n. ${ }^{\circ}$ 16: 469-470.

Cable News Network-CNN México. 2015. "La libertad de expresión no da derecho a insultar la fe de otros". CNN México, 15 de enero. Acceso el 06 de abril de 2016. http:// mexico.cnn.com/mundo/2015/01/15/lalibertad-de-expresion-no-da-derecho-ainsultar-la-fe-de-otros-papa

De la Corte, Luis; Kruglanski, Arie; Díaz, Darío y Sabucedo, José Manuel. 2007. "Siete principios psicosociales para explicar el terrorismo". Psicothema 19, n. ${ }^{\circ} 3: 366-374$.
Díaz, Fernando. 2007. "Trauma Colectivo y Terrorismo". Umbral Científico 10: 133148. http://www.redalyc.org/articulo. oa? id=30401011

FOX NEWS. 2003. "Timeline: Sept. 11, 2001". FOX NEWS, 11 de setiembre. Acceso el 06 de febrero de 2015. http://www.foxnews.com/story/2003/09/11/timelinesept-11-2001/

Indo Memorial Site. 2003. "Remember Bali. Memorial Site". Acceso el 06 de febrero de 2015. http://www.indo.com/ bali121002/

Krueger, Alan y Mal ková, Jitka. 2003. "Education, Poverty and Terrorism: Is there a causal conection?". Jorunal of Economic Perspectives 17, n. ${ }^{\circ}$ 4: 119-144.

Labari, N. 2004. "Millones de personas se manifiestan contra el terrorismo". El Mundo, 13 de Marzo. Acceso el 06 de abril de 2016. http://www. elmundo.es/elmundo/2004/03/12/ espana/1079113316.html

Mercado, Asael; González, Guillermo y Olvera, Jorge. 2009. "La crisis del orden mundial: Globalización y terrorismo”. Revista de Relaciones Internacionales, Estrategia y Seguridad 4, n. ${ }^{\circ}$ 1: 129-158.

El Mercurio. 2001. "Especiales EMOL 11-S. El ataque que aterrorizó al mundo". El Mercurio. Acceso el 06 de febrero de 2015. http://www.emol.com/ especiales/11s_2006/01_losatentados_.htm

El País. 2003. "El atentado de Casablanca deja 41 muertos". El País, 18 de mayo. Acceso el 06 de febrero de 2015. http:// elpais.com/diario/2003/05/18/internacional/1053208801_850215.html

Pavon, Victor y Velázquez, Jorge. 2006. “Es la pobreza la causa del terrorismo?". Foro Internacional XLVI, n. 2 (abril-junio): 291-302.

Rodríguez, Tania Graciela. 2012. "El terrorismo y nuevas formas de terrorismo". Espacios Públicos 15, n. 33 (enero): 72-95.

Romero, Antonio José y Durán, María del Mar. 2010. "Islam y terror". Convergencia. Revista de Ciencias Sociales 17, n. ${ }^{\circ}$ 54: 53-68. 
Sánchez, Gabriel. 2005. "Informar sobre terrorismo: una misión difícil pero necesaria”. Comunicación y Hombre 1: 71-89.

Sequera, Norma. 2001. "Terrorismo y educación”. Educere 5, n. ${ }^{\circ} 15: 311-317$.

Stelter, Brian. 2015. "Charlie Hebdo exageró con sus caricaturas, asegura cofundador". CNN México, 17 de enero. Acceso el 06 de abril de 2016. http://mexico.cnn. com/mundo/2015/01/17/charlie-hebdoexagero-con-sus-caricaturas-asegura-uncofundador

Vázquez, José Carlos. 2012. "La racionalidad del mexicano desde la teoría amplia Elsteriana". Revista Internacional de Ciencias Sociales y Humanidades XXII, n. ${ }^{\circ} 1$ : 249-262.

Vázquez, José Carlos. 2015. "Las creencias como proceso de readaptación social. Una aproximación desde la perspectiva de la teoría eslteriana de la racionalidad". Revista Nueva Antropología 27, n. ${ }^{\circ} 80$ : 139-150.

Zuinaga, Soraya. 2011. "El terrorismo, una aproximación teórica en cuanto a su definición". Revista Venezolana de Análisis de Coyuntura xvII, n. ${ }^{\circ} 2$ (juliodiciembre): 11-26.

\section{TEXTOS ELECTRÓNICOS}

Real Academia de la Lengua Española (RAE). 2015. Real Academia de la Lengua Española. Acceso el 06 de febrero de 2015. http://www.rae.es/

\section{OTROS DOCUMENTOS}

Galimberti, U. 2002. Diccionario de psicología. Ciudad de México: Siglo XxI Editores.

Oficina del Alto Comisionado de las Naciones Unidas para los Derechos Humanos. 2008. Folleto informativo No. 32. Los Derechos Humanos, el Terrorismo y la Lucha contra el Terrorismo. Naciones Unidas. Ginebra.

Fecha de ingreso: 08/10/2015 Fecha de aprobación: 15/02/2016 